\title{
Comparison of Peripherally Inserted Central Venous Catheter (PICC) versus Central Venous Catheter (CVC): A Consecutive Selection Study
}

\author{
M Hubrich², K Lundholm¹ , J Snygg², S Lindgren², C Engström ${ }^{1}$ \\ ${ }^{1}$ Department of Surgical Science, The Sahlgrenska Academy, Gothenburg University, Sweden \\ ${ }^{2}$ Dept of Anaesthesiology and Intensive Care Medicine, The Sahlgrenska Academy,
}

\section{BACKGROUND}

Gothenburg University, Sweden.

The safest and most cost efficient choice of central venous access is still a matter of concern in clinical care and research. A safe and long lasting central venous catheter (CVC) with a low number of side effects is preferred. Few clinical trials have been published comparing complications and patency of CVC:s and peripherally inserted catheters (PICC:S). (1-4) We performed a consecutive selection study on patients referred to the Department of Surgery at Sahlgrenska University Hospital, randomized to either PICC or CVC.

\section{METHODS}

In 2014-15, 155 patients admitted for upper gastrointestinal cancer surgery and in need of a CVC/PICC were enrolled. End-points were development of complications requiring catheter removal, catheter related infection, thrombophlebitis, pneumo- and hemothorax. Anaesthesiologists performed CVC insertions. PICC:s were inserted by a register nurse or anesthesiologist. A study protocol followed the patients where baseline-data, catheter days, patient experience and complications were registered. (Figure 1, Table 1)

\section{RESULTS}

114 patients received a CVC and 42 a PICC. No difference between treatment groups was found regarding gender, age, BMI, associated diagnosis, antibiotic, antitrombotic or corticosteroid treatment at postop day one. However, days of treatment reached significant difference, with more days in the PICC line (21 days) vs. CVC ( 15 days) group, $p<0.05$. The number of leukocytes was significantly higher in PICC patients (10.1) compared to CVC patients (7.9), $p<0.005$. Accordingly, the CRP sample results in the PICC group were higher (51.0) compared to the CVC group (16.3).

\begin{tabular}{|c|c|c|c|}
\hline \multicolumn{4}{|c|}{ Table 1. Reason for Catheter removal CVC vs. PICC } \\
\hline Intention to treat (all) & CVC & PICC & $\mathrm{p}<$ \\
\hline end of treatment & 81 & 25 & ns \\
\hline death & 2 & 1 & ns \\
\hline change of catheter & 4 & 4 & ns \\
\hline complication & 9 & 4 & $\mathrm{~ns}$ \\
\hline other or unknown & 16 & 3 & ns \\
\hline \multicolumn{4}{|l|}{$\begin{array}{l}\text { Per protocol (followed } \\
\text { randomisation) }\end{array}$} \\
\hline end of treatment & 56 & 18 & ns \\
\hline death & 2 & 1 & ns \\
\hline change of catheter & 3 & 4 & ns \\
\hline complication & 7 & 1 & ns \\
\hline other or unknown & 8 & 3 & ns \\
\hline
\end{tabular}

\section{CONCLUSION}

- We found no statistically significant difference regarding complications or patient comfort during the time period of catheter treatment, which was significantly longer with PICC than CVC.

- The results of this study imply that the choice of CVC or PICC in this study population should be based only upon the potential duration of treatment postoperatively.
Table 1

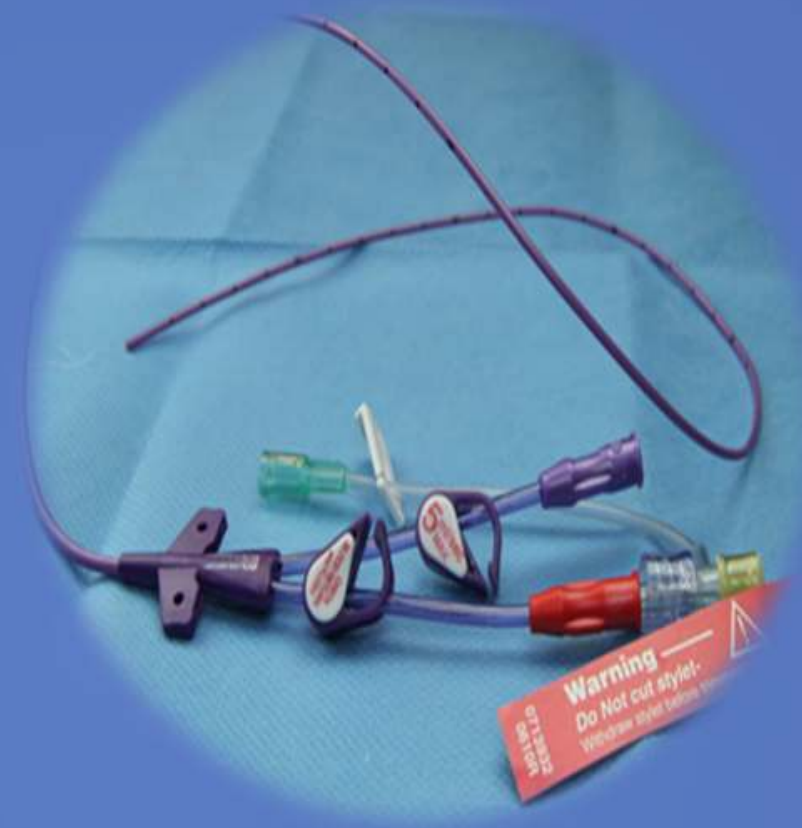

RESULTS (cont.)

However there were not significantly more infections at time of insertion among the PICC line treated patients. We found very few local infections or septic complications over all. We did not find any difference in catheter comfort between treatment groups. (Table 1, 2, 3)

Table 2 Table 2. Catheter treatment experience from patients CVC vs. PICC

\begin{tabular}{|l|l|l|l|}
\hline Intention to treat (all) & CVC & PICC & $p<$ \\
\hline None & 65 & 14 & ns \\
\hline Milld to moderate & 13 & 2 & ns \\
\hline Severe & 1 & 2 & ns \\
\hline Unknown & 33 & 19 & ns \\
\hline & & & \\
\hline $\begin{array}{l}\text { Per protocol (followed } \\
\text { randomisation) }\end{array}$ & & & \\
\hline None & 45 & 13 & ns \\
\hline Mild to moderate & 8 & 2 & ns \\
\hline Severe & 0 & 1 & ns \\
\hline Unknown & 21 & 10 & ns \\
\hline & & & \\
\hline
\end{tabular}

\begin{tabular}{|c|c|c|c|}
\hline & CVC & PICC & $p<$ \\
\hline \multicolumn{4}{|l|}{ Intention to treat (all) } \\
\hline none & 84 & 27 & ns \\
\hline local infection & 6 & 1 & ns \\
\hline occlusion & 10 & 3 & ns \\
\hline unsuccessful insertion & 1 & 1 & ns \\
\hline pneumonia & 1 & 1 & ns \\
\hline septicemia & 1 & 0 & ns \\
\hline thrombophlebitis & 1 & 1 & ns \\
\hline hemo-/pneumothorax & 0 & 0 & ns \\
\hline other & 8 & 3 & ns \\
\hline \multicolumn{4}{|c|}{ Per protocol (followed randomisation) } \\
\hline none & 56 & 20 & ns \\
\hline local infection & 3 & 1 & ns \\
\hline occlusion & 6 & 1 & ns \\
\hline unsuccessful insertion & 1 & 1 & ns \\
\hline pneumonia & 1 & 1 & ns \\
\hline septicemia & 1 & 0 & ns \\
\hline thrombophlebitis & 1 & 1 & ns \\
\hline hemo-/pneumothorax & 0 & 0 & ns \\
\hline other & 7 & 2 & ns \\
\hline
\end{tabular}

